\title{
INTERFERÊNCIA LINGUÍSTICA DO INGLÊS EM ESTUDANTES DE ESPANHOL. O CASO DE ESTUDANTES UNIVERSITÁRIOS NA ESPANHA
}

\author{
INTERFERENCIA LINGÜÍSTICA DEL INGLÉS EN ESTUDIANTES CHINOS \\ DE ESPAÑOL. EL CASO DE ESTUDIANTES UNIVERSITARIOS EN ESPAÑA
}

\author{
LINGUISTIC INTERFERENCE OF ENGLISH IN CHINESE STUDENTS OF \\ SPANISH. THE CASE OF UNIVERSITY STUDENTS IN SPAIN
}

Carles NAVARRO-CARRASCOSA ${ }^{1}$

\begin{abstract}
RESUMO: Este trabalho apresenta um estudo sobre a interferencia lingüística entre estudantes chineses de inglês e de espanhol. Em especial se investiga a influencia que pode ter exercido o inglês como L2, no espanhol como L3. Para realizar esta investigação, se utiliza uma amostragem formada por um grupo de estudantes de E/LE chineses que está realizando um ano pré-universitário na Espanha e se comparam suas produções com um grupo de estudantes universitarios americanos que também estuda espanhol (neste caso, como L2), que estudaram um semestre neste mesmo país. Comparam-se os erros de ambos grupos para extrair dados que permitem conhecer quais as influências do inglês e o grau das influências desta língua no aprendizado de E/LE por estudantes sinofalantes.
\end{abstract}

PALAVRAS-CHAVE: Interferência linguística. Sinofalantes. E/LE. Análise de error.

RESUMEN: En el presente trabajo se plantea un estudio sobre la interferencia lingüística en estudiantes chinos de inglés y de español. Especialmente se busca la influencia que puede haber tenido el inglés, como L2, en el español, como L3. Para realizar la investigación, se ha tomado como muestra un grupo de estudiantes de E/LE chinos que están realizando un año preuniversitario en España y se han comparado sus producciones con un grupo de universitarios estadounidenses, también estudiantes de español (aunque en este caso esta lengua es la L2), que realizaron una estancia de un semestre en el mismo país. Se han comparado los errores de unos y otros para poder extraer cuáles son por influencia del inglés y el grado de influencia de esta lengua en el aprendizaje de E/LE de los sinohablantes.

PALABRAS CLAVE: Interferencia lingüística. Sinohablantes. E/LE. Análisis de errores.

ABSTRACT: In the present work, a study about linguistic interference in Chinese students of English and Spanish is proposed. Especially looking for the influence that English may have had, such as L2, in Spanish, as L3. To carry out the research, we

${ }^{1}$ Universitat de València (UV), Valencia - España. Profesor del departamento de Filología Española. ORCID: https://orcid.org/0000-0002-0303-982X. E-mail: carles.navarro@uv.es 
have taken as a sample a group of Chinese E/LE students who are doing a preuniversity year in Spain and have compared their productions with a group of American university students, also Spanish students (although in this case this language is L2), who made a one-semester stay in the same country. The errors of each one have been compared to be able to extract which ones are influenced by English and the degree of influence of this language in the learning of E/LE of the syllables.

KEYWORDS: Linguistic interference. Chinese learners. SFL. Error analysis.

\section{Introducción}

Cualquier docente de E/LE que haya tenido una clase con estudiantes de diversas nacionalidades, entre las cuales se encontrara algún discente chino, habrá apreciado que el ritmo de aprendizaje es más lento en el sinohablante que en el resto del alumnado. Sucede los mismo con otros estudiantes asiáticos. Esto es así hasta tal punto que muchas veces, especialmente en los cursos intensivos, a este estudiante hay que bajarlo de nivel para que el resto pueda seguir su progreso y él o ella pueda dedicar el tiempo que necesite a su aprendizaje.

El motivo no es otro que la mayor distancia que hay entre la lengua materna de estos estudiantes y el sistema lingüístico meta, en este caso el español. La mayoría de lenguas occidentales, aunque sean de familias diferentes, suelen tener más similitudes entre sí que la mayoría de las lenguas asiáticas.

Por eso, no es muy diferente cuando el grupo está compuesto íntegramente por estudiantes de China, pues la diferencia entre ambos sistemas lingüísticos (el materno y el meta) es la misma. La única ventaja que se puede dar es que todos los estudiantes necesitarán un mismo ritmo y quizás la frustración, consecuencia del lento ritmo de aprendizaje, será menor o, incluso, inapreciable.

Por esta distancia que hemos mencionado entre el sistema lingüístico chino y el español, los estudiantes de E/LE sinohablantes se acogen a estructuras lingüísticas de otros sistemas que ya hayan estudiado y que sean más similares al de la lengua meta. Es lo que sucede con el inglés, que a pesar de no ser una lengua románica, tiene (o puede parecer que tenga) más similitudes con el español de las que tendrá el chino mandarín.

Esta investigación no se centra en la enseñanza, sino más bien en el aprendizaje de estudiantes sinohabantes de español como lengua extranjera (E/LE). En específico, buscamos saber hasta qué punto las lenguas que estos estudiantes han estudiado previamente (en concreto, el inglés) influyen en la adquisición de E/LE. 
Para este estudio, hemos trabajado en paralelo con dos grupos de estudiantes diferentes:

1. Por un lado, un grupo de estudiantes universitarios de los Estados Unidos que realizaron una estancia en la Universitat de València (España) durante los meses de enero a mayo de 2019. Estos estudiantes tenían entre 20 y 23 años de edad, su lengua materna es el inglés y tenían un nivel B1 de español cuando empezaron el curso.

2. El segundo grupo de informantes para este estudio era el verdadero foco de la investigación. Dieciocho estudiantes chinos, procedentes de BLCU (Beijin Language and Culture University) y que llegaron a Valencia para estudiar español durante un semestre antes de ingresar en distintas universidades españolas. Estos estudiantes tenían entre 19 y 23 años en el momento del estudio, su lengua materna es el chino mandarín y su nivel de español estaba entre el A2 y el B1. Todos ellos habían estudiado inglés previamente y afirmaron que el inglés es su L2 y el español, su L3. Este grupo de estudiantes llevan desde enero de 2019 estudiando español en el área de idiomas de ESIC Business \& Marketing School, en Valencia.

\section{La interferencia linguística}

Isabel Santos Gargallo (1999, p. 28) dice que el proceso de enseñanzaaprendizaje de una lengua es un proceso gradual por el que los aprendientes adquieren ciertos mecanismos de comunicación. Dicho proceso está

constituido por etapas, con un punto de partida (L1) y un punto de llegada (L2). Cada una de esas etapas entre L1 y L2 son estadios de aprendizaje que constituyen lo que llamamos Interlengua [...]. Es un sistema que posee rasgos de la lengua materna, de la lengua meta y otros propiamente idiosincrásicos, y cuya complejidad se va incrementando en un proceso creativo que atraviesa sucesivas etapas marcadas por los nuevos elementos que el hablante interioriza.

El término Interlengua (IL) fue acuñado por Selinker en 1972 para referirse al sistema lingüístico en construcción, ese lenguaje de los hablantes no nativos que no han terminado de aprender la lengua meta. A lo largo del proceso de aprendizaje, el idioma que se estudia pasa por diferentes fases, cada una más evolucionada que la anterior. Estas fases representan las distintas etapas de la IL, que quedan muy bien representadas en el siguiente esquema: 
Esquema 1: Distintas fases de la IL.

$$
\mathrm{L}_{1} \rightarrow \mathrm{IL}_{1} \ldots \mathrm{IL}_{2} \ldots \mathrm{IL} 3 \ldots \mathrm{IL} 4 \ldots \mathrm{IL} n \rightarrow \mathrm{L}_{2}
$$

Fuente: Santos Gargallo (1972, p. 28).

En relación a la IL, surge otro cocepto interesante para esta investigación: la transferencia. Este término tiene su origen en el conductismo y su propuesta de aprendizaje a través las asociaciones de estímulos y respuestas. Desde el punto de vista del aprendizaje de lenguas, la tranferencia es el uso "automático e inconsciente de conductas ya adquiridas en nuevas situaciones de aprendizaje" (ALONSO GARCÍA, 2003, p. 9). La transferencia es, por tanto, el calco de una estructura, léxica o gramatical, de una lengua a otra. Puede ser positiva, si el calco beneficia al uso correcto en la L2, o negativa, si lo que hace es entorpecer el uso correcto como consecuencia de diferencias presentes entre ambas lenguas. La transferencia negativa desemboca en errores y recibe el nombre de interferencia.

En el presente trabajo no se buscan los errores producidos por la interferencia del chino en el español, sino aquellos que produce la interfernecia del inglés (L2) en el español (L3) en estudiantes cuya lengua materna es el chino mandarín (L1).

Imagen 1 - Interferencia lingüística de la L2 en la interlengua hacia la L3

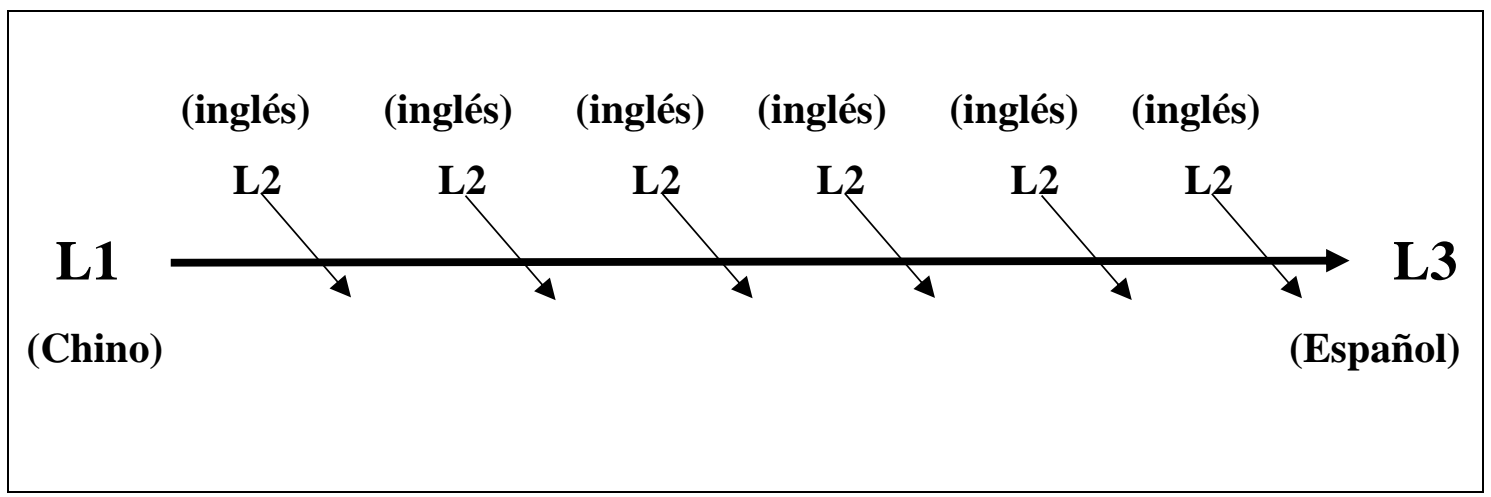

\section{Particularidades de la lengua china}

Para poder descartar qué errores no se derivan de la interferencia de la lengua materna del grupo de estudiantes sinohablantes, es importante entender cómo funciona el sistema lingüístico chino.

Para ello, hay varias obras y estudios descriptivos que se pueden consultar. Destacan los trabajos de Cortés Moreno (2002, 2009, 2014), el de Liu (2008) y el 
manual de escritura y gramática de Rovira (2017). En España se han publicado varias obras desde el ámbito universitario sobre la lengua china, especialmente dedicadas a la traducción, como la obra de dos volúmenes de Casas, Rovira y Suárez (2015). Sin dejar de hacer especial referencia a la obra gramatical, publicada en 2015 por Po-Ching y Rimmington, que es una gramática de la lengua china, íntegramente en español.

Una de las primeras particularidades del chino mandarín es que sus palabras no tienen variables de género ni de número. Esto no quiere decir que no exista el género, el número, el tiempo, el modo y la persona (rasgos lingüísticos que en español vienen marcados por determinados morfemas o marcas lingüísticas), pero en chino no están marcados por indicadores, necesariamente. Por lo tanto, para poder saber, por ejemplo, si una palabra es masculina o femenina o el número de la misma, entre otras cosas, habrá que atender al contexto comunicativo o al contorno lingüístico próximo.

El orden sintáctico de base es muy similar al del español (sujeto + verbo + objeto). Sin embargo, las diferencias entre ambos sistemas se van haciendo más evidentes en cuanto se van añadiendo elementos al sintagma oracional:

-Si hay elementos que indican tiempo y lugar, el orden oracional pasa a ser: sujeto + tiempo + lugar + verbo + objeto.

-Si hay un sintagma preposicional, este aparece siempre delante del verbo.

-Las construcciones de genitivo también se anteponen al verbo.

-El adjetivo se antepone al sustantivo.

-En ese sentido, es lógico que las oraciones de relativo se coloquen antes de los sustantivos a los que hacen referencia, y no después, como sucede en español.

-La comparación referida a un adjetivo va precedida a dicho adjetivo.

Otra curiosidad es que en chino, a pesar de que existen las preposiciones, hay preferencia por las postposiciones. Esto quiere decir que es habitual que estas partículas sintagmáticas aparezcan después del elemento al que acompañan. Dos ejemplos ilustrativos serían los que aparecen el la tabla 1:

Tabla 1 - Comparación con el sistema preposicional y una simulación del sistema posposcional

\begin{tabular}{|c|c|}
\hline Sistema preposicional & Sistema posposicional \\
\hline Sobre la silla & $*$ Silla sobre \\
\hline Dentro del coche & $*$ Coche interior \\
\hline
\end{tabular}


Otra consideración importante que hay que hacer sobre las diferencias entre la lengua española y la china es que en esta última hay una tendencia a frases de sintagma adjetivo en vez de oraciones subordinadas. Por ejemplo:

(1) El libro que María ha escrito

*El-por-María-escrito libro

(2) El debate del que la gente habla

*Habla-la-gente debate

\section{Metodología}

Como el objetivo de este estudio es comprobar si la L2 (el inglés) tiene influencia en la L3 (el español) en los estudiantes sinohablantes, fue decisivo comparar los errores que se repetían en los dos grupos de estudiantes: los estadounidenses y los chinos.

Para ello, se les pidió a ambos grupos que realizaran escritos de la misma temática, considerando que, aunque los contenidos fueran diferentes, la misma temática les invitaría a tomar estructuras gramaticales y léxicas similares. Para que cada composición tuviera output suficiente para poder ser analizada, debían tener entre 250 y 300 palabras.

Imagen 2 - Enunciado de la redacción que se les propuso a ambos grupos

Hay muchas personas que están contentas con el lugar donde viven y hay otras muchas que siguen soñando con encontrar un lugar para vivir.

Escribe una redacción sobre este tema. En ella tienes que:

-describir las características que debe tener el lugar perfecto para vivir;

-hablar del entorno: clima, paisaje, servicios, etc.;

-explicar con quién te gustaría compartir ese lugar.

A través de las indicaciones que se les ofreció a los estudiantes para que produjeran sus escritos, se pretendía, como ya se ha señalado, que ambos grupos requirieran de formas gramaticales y léxicas similares, de forma que pudiera compararse si las estructuras que fueran producto de la interferencia lingüística con el inglés en los 
estudiantes estadounidenses serían las mismas en los estudiantes chinos y, de esta manera, comprobar si esas estructuras en los estudiantes sinohablantes eran influencia del inglés.

Al pedirles una descripción de un lugar se esperaba que todos los estudiantes de los dos grupos utilizaran vocabulario relacionado con el entorno, tanto urbano como rural; pidiéndoles las características del lugar perfecto se pretendía que se sintieran libres y creativos para elegir el lugar en el que realmente les gustaría vivir y cómo les gustaría que fuera, en cualquier aspecto; al insistir en la descripción (con el segundo punto) se pretendía que no dejaran de hablar de una serie de cosas $\mathrm{y}$, de este modo, asegurarnos que todos los informantes utilizaban vocabulario relacionado; al pedirles que plasmaran en el papel con quién les gustaría compartir ese lugar, pretendíamos que tuvieran que introducir personas de su entorno y deseos de futuro sobre sus propias relaciones personales.

Todos estos criterios les obligarían a utilizar el vocabulario del entorno, el clima, la ciudad, etc., el de las relaciones personales (tanto familiares, como de otro tipo), así como utilizar estructuras gramaticales de futuro, a formular algunas hipótesis y a utilizar estructuras de deseo (con o sin presente de subjuntivo).

Además, como el foco de estudio era el grupo de sinohablantes, se preparó un cuestionario especialmente para ellos, en el que se pretendía extraer información a cerca de sus conocimientos de inglés y de español y la influencia de la primera en su interlengua hacia la segunda, es decir, la influencia que sus conocimientos del inglés tenían en sus producciones en español.

En el cuestionario, después de varias preguntas relacionadas con su nivel de ambas lenguas, se les pedía que escribieran frases de dificultad progresiva en inglés y que, posteriormente, las tradujeran al español. Por ejemplo:

(1) Write a sentence in which you describe yourself in English

(2) Write a sentence in which you describe a friend in English

Así, hasta llegar a peticiones que exigieran la producción de oraciones más complejas:

(3) You won the lottery and you've spent all your money on holidays and whims. Write a sentence wishing to go back and do things differently 
Con este ejercicio se pretendía, por un lado, comprobar en cuál de las dos lenguas el estudiante tenía un nivel más alto; por otro, que los informantes hicieran las mismas producciones tanto en la L2 como en la L3 para poder comparar qué estructuras de uma lengua interferían en la otra.

\section{Resultados}

El número de estudiantes de los dos grupos de que nos han servido como informantes (el grupo de estudiantes estadounidenses y el grupo de estudiantes chinos) representan muestras pequeñas para realizar un análisis cuantitativo. Sin embago, vamos a presentar una serie de porcentajes que hemos extraído de los errores que ha habido en las redacciones de ambos grupos como consecuencia de la interferencia lingüística. No obstante, estos porcentajes tendrán valores estrictamente orientativos.

Los errores ocasionados por la interferencia lingüística que más presencia han tenido en ambos grupos los hemos clasificado en las siguientes categorías:

Tabla 2 - Porcentajes de los distintos errores de interferencia lingüística

\begin{tabular}{|c|c|c|}
\hline Error & Estadounidenses anglófonos & Sinohablantes \\
\hline Conjunciones & $7,89 \%$ & $15,87 \%$ \\
\hline Preposiciones & $35,53 \%$ & $6,35 \%$ \\
\hline Concordancia & $34,21 \%$ & $30,16 \%$ \\
\hline Léxico & $3,95 \%$ & $25,40 \%$ \\
\hline Verbos & $15,79 \%$ & $15,87 \%$ \\
\hline Otros errores & $2,63 \%$ & $6,35 \%$ \\
\hline
\end{tabular}

La diferencia de errores en algunas categorías no tiene por qué ser significativa para el estudio, pues no necesariamente se debe a la transferencia negativa del inglés. Hay muchos factores que se han tenido en cuenta para el análsis, como la diferencia de nivel (aunque es similar en ambos grupos, hay estudiantes sinohablantes que están un poco por debajo de sus compañeros) o la lengua materna de cada grupo, que puede hacer que unos errores tengan más presencia en un grupo que en otro.

Por tanto, este análisis aumentará su significatividad si analizamos de forma más minuciosa cada una de las categorías de error que hemos encontrado.

Las conjunciones, como hemos visto en la tabla anterior, son un error más frecuente en el grupo de sinohablantes que en el de estadounidenses. No obstante, los 
porcentajes en los errores no coinciden en ambos grupos, como veremos en la siguiente tabla:

Tabla 3 - Porcentaje de errores en las conjunciones.

\begin{tabular}{|c|c|c|}
\hline Error & Estadounidenses anglófonos & Sinohablantes \\
\hline Confusión so> así que & $16,67 \%$ & $0 \%$ \\
\hline Ausencia de que subordinado & $16,67 \%$ & $70 \%$ \\
\hline Relativos que/quien & $33,33 \%$ & $0 \%$ \\
\hline Aparición de que incorrecta & $33,33 \%$ & $30 \%$ \\
\hline
\end{tabular}

El primero de los errores de esta categoría, la confusión entre el valor final y el explicativo de so en inglés es un error típico de los anglófonos en español, como podemos apreciar en el ejemplo extraído de una de las estudiantes estadounidenses:

(4) *Me gustaría tener un jardín así que yo puedo cultivar

Sería esperable que los estudiantes chinos cometieran el mismo error por influencia negativa del inglés, sin embargo no se ha dado ningún caso en las producciones que hemos recopilado.

Otro de los errores es la confusión entre los relativos que y quien; error más frecuente en anglófonos, por interferencia de los distintos usos que hay en inglés de sus respectivos equivalentes (that y who).

Sobre la ausencia de la conjunción subordinada sustantiva que, es un error de interferencia que se repite en ambos grupos, especialmente en los sinohablantes. En los anglófonos es un claro error de influencia negativa del inglés:

\section{(5) *Yo era la única hija hasta mi hermano nació}

I was the only daughter until my brother was born

Cabría pensar que el error en los sinohablantes podría venir por influencia del inglés, donde esta conjunción no es necesaria cuando hay otro elemento de unión, como las preposiciones. No obstante, el chino mandarín es una lengua en la que las construcciones sustantivas y adjetivas no se hacen por medio de nexos, sino a través de sintagmas más elaborados, como ya vimos más arriba. Por eso, es cuestionable que ejemplos de output del grupo de estudiantes sinohablantes, como: 
(6) *Deseo cerca de la ciudad donde vivo hay una montaña

(7) *Hay una universidad y un hospital están cerca de mi casa

sea por interferencia del inglés y no de la propia lengua china, que sería la L1, en este caso.

Otro de los errores que se esperaría de estudiantes de E/LE que tienen un elevado nivel de inglés como L2 sería el que se encuentra a veces en estudiantes de español cuya lengua materna es el inglés; se trata de la confusión de la preposición to, que suele acompañar al verbo en infinitivo y que muchas veces introduce oraciones subordinadas sustantivas de infinitivo. El error consiste en traducir este to por el nexo que, pensando que con el infinitivo también se necesita una conjunción, al igual que en inglés:

(8) *Yo escogería que vivir en Illinois

Este error se ha encontrado en muchas ocasiones en los estudiantes chinos de ELE:

(9) *Es importante que vivir en el lugar

(10) *El lugar necesita que está tranquilo

Como hemos explicado más arriba, las construcciones subordinadas en el chino mandarín se hacen a través de sintagmas, por lo que sí que podemos considerar que, en este caso, el uso incorrecto del nexo subordinado que es por interferencia del inglés.

Los siguientes errores por transferencia negativa que comentaremos son aquellos relativos al usos de las preposiciones:

Tabla 4 - Porcentaje de errores en las preposiciones

\begin{tabular}{|c|c|c|}
\hline Error & Estadounidenses anglófonos & Sinohablantes \\
\hline Aparición incorrecta & $22,22 \%$ & $50 \%$ \\
\hline Ausencia incorrecta & $14,81 \%$ & $25 \%$ \\
\hline Confusión preposiciones & $40,74 \%$ & $25 \%$ \\
\hline Por/para & $18,52 \%$ & $0 \%$ \\
\hline Colocación incorrecta & $3,71 \%$ & $0 \%$ \\
\hline
\end{tabular}

Antes de empezar a analizar los resultados de nuestro corpus, hay que comentar dos cuestiones: la primera es que, como ya se mencionó en su respectivo apartado, el chino mandarín tiene preferencia por la posposición frente a la preposición, por lo que 
tiene sentido que los estudiantes sinohablantes tegan facilidad para no equivocarse en el orden de la colocación de las partículas españolas una vez han aprendido que siempre van antepuestas al sustantivo al que acompañan; la segunda es el hecho de que no haya aparecido ningún error en lo que se refiere a las preposiciones por y para, que se debe a la tendencia que tienen los sinohablantes de traducir los nexos de causa y consecuencia chinos por las locuciones conjuntivas por lo que, debido a, como y entonces.

Sobre la aparición y la ausencia incorrectas de preposiciones, ya señala Cortés (2009) que tiene más relación con la interferencia con el inglés que con la propia L1. Y sobre la confusión de unas por otras, el mismo autor indica que "dominar las preposiciones españolas no les resulta fácil a los sinohablantes" $(2009$, p. 179).

Los errores de concordancia que encontramos en ambos grupos, pueden ser de dos tipos: error de concordancia de género entre el significante y el referente; y error de concordancia de género y número entre un sustantivo y el adjetivo que lo acompaña.

Tabla 5 - Porcentaje de errores de concordancia

\begin{tabular}{|c|c|c|}
\hline Error & Estadounidenses anglófonos & Sinohablantes \\
\hline $\begin{array}{c}\text { Género entre significante y } \\
\text { referente }\end{array}$ & $11,54 \%$ & $15,79 \%$ \\
\hline $\begin{array}{c}\text { Género y número entre } \\
\text { adjetivo y sustantivo }\end{array}$ & $88,46 \%$ & $84,21 \%$ \\
\hline
\end{tabular}

Los datos son muy similares en ambos grupos. En el primer tipo de error, que se refiere a una fallo de concordancia de género entre el lexema y el referente, el fallo se da siempre con el género del emisor, es decir, si el emisor es una mujer, el género del lexema se construirá en femenino, independientemente del género del referente:

(11) *[Él] estaba constantemente preocupada por su familia (emisor mujer).

(12) *[Él] estaba muy emocionada (emisor mujer).

Por ello, parece más un error de fosilización sin relación con la L1, en el caso de los estudiantes estadounidenses, ni con la L2, en el caso de los estudiantes chinos.

Las interferencias léxicas son más llamativas. Se dan en ambos grupos y la estructura del significante hace más evidente que la causa es el inglés: 
Tabla 6 - Porcentaje de errores en el léxico

\begin{tabular}{|c|c|c|}
\hline Error & Estadounidenses anglófonos & Sinohablantes \\
\hline conveniencia por comodidad & $66,67 \%$ & $81,25 \%$ \\
\hline hacer por tomar & $33,33 \%$ & $0 \%$ \\
\hline confortable por cómodo & $0 \%$ & $6,25 \%$ \\
\hline moverse por mudarse & $0 \%$ & $6,25 \%$ \\
\hline sentencia por frase & $0 \%$ & $6,25 \%$ \\
\hline
\end{tabular}

La adaptación del lexema inglés al sistema castellano por parte de los estudiantes sinohablantes en ejemplos como:

(13) *Hay muchas tiendas de comestibles cerca por conveniencia

(14) *Enseñar en la misma escuela de mis niños o cerca para ser conveniente

(15) *El transporte es muy conveniente

(16) *Es muy conveniente para mí ir al médico

(17) *Estoy escribiendo una sentencia

(18) *El clima confortable

nos hacen ver con claridad la interferencia lingüística que se ha dado en ellos. Estos son solo algunos ejemplos representativos que se han dado en los escritos (también en algunas de las encuestas que no han sido contabilizados en estos porcentajes), por lo que hay que entender que si se aumenta la muestra aparecerán muchos más ejemplos. Destacamos el ejemplos de conveniencia, que es el que más abunda en el caso de los sinohablantes y el único que se repite en los dos grupos.

Los errores relacionados con el sistema verbal también se pueden deber a varios factores, como veremos en la siguiente tabla:

Tabla 7 - Porcentaje de errores en el sistema verbal.

\begin{tabular}{|c|c|c|}
\hline Error & Estadounidenses anglófonos & Sinohablantes \\
\hline Ser y estar & $33,34 \%$ & $7,14 \%$ \\
\hline Subjuntivo & $8,33 \%$ & $28,58 \%$ \\
\hline Gerundio calcado & $33,34 \%$ & $0 \%$ \\
\hline Infinitivos & $8,33 \%$ & $14,28 \%$ \\
\hline Construcciones pasivas & $8,33 \%$ & $0 \%$ \\
\hline Presente continuo & $8,33 \%$ & $0 \%$ \\
\hline Omisión de verbos & $0 \%$ & $28,58 \%$ \\
\hline Hay $<$ There is/There are & $0 \%$ & $21,42 \%$ \\
\hline
\end{tabular}


La confusión entre los verbos ser y estar y el subjuntivo son errores esperables en ambos grupos, ya que tanto el inglés como el chino son lenguas muy diferentes del español y sus sistemas verbales distan en estos aspectos del de la lengua de Cervantes.

Los estudiantes chinos y los estadounidenses parecen tener errores propios que no comparten con el otro grupo. Así, los anglófonos cometen fallos en el mal uso del gerundio en estructuras calcadas del inglés (Alonso García, 2003), entre las que podemos incluir la construcción estar + gerundio o presente continuo, y en el abuso de las construcciones pasivas, que son muy frecuentes en la lengua inglesa; los sinohablantes, por otro lado, se hallan con problemas para utilizar correctamente la forma impersonal hay y omitir algunos verbos. Sobre esto último, es frecuente en el chino mandarín la omisión verbal si la acción se puede interpretar por el contexto y se han encontrado influecias de esto en español:

(19) * Me gusta mucho [ir] de compras con mi novia

(20) *Quiero un lugar para vivir que debe [ser] más bonito

Sobre el impersonal hay para expresar existencia, sí que percibimos la influencia negativa del inglés en algunos casos (es positiva en muchos otros), como en:

(21) *Siempre hay el sol (< There is always the sun)

Este ejemplo es una traducción directa del inglés que parece no darse en los estudiantes anglófonos.

Hay otros errores por transferencia negativa en ambos grupos:

Tabla 8 - Porcentaje de errores en las preposiciones

\begin{tabular}{|c|c|c|}
\hline Error & Estadounidenses anglófonos & Sinohablantes \\
\hline Adjetivación de sustantivos & $50 \%$ & $50 \%$ \\
\hline Anteposición de adjetivos & $50 \%$ & $50 \%$ \\
\hline
\end{tabular}

Solo han aparecido dos errores en cada caso en ambos grupos, por lo que parecen no ser recurrentes. No obstante, se dan en ambos tipos de aprendientes y en ambos casos son interferencias de las lenguas maternas de cada grupo de estudiantes. En inglés es habitual que los adjetivos vayan pospuestos al sustantivo, igual que en chino mandarín. Sobre la adjetivación de sustantivos, en ambas lenguas los adjetivos pueden 
coincidir en forma con los sustantivos. La categoría gramatical del lexema se sabe por el contexto o por el contorno oracional.

Es posible que los estudiantes sinohablantes tengan un refuerzo de dicha estructura por sus conocimientos linguiísticos del inglés, pero no podemos hablar de interferencia.

\section{Consideraciones finales}

Para concluir esta investigación, se puede afirmar que los estudiantes chinos tienen influencia de la lengua inglesa, tanto positiva como negativa. Las influencias negativas o interferencias del inglés en el chino se dan, en el caso que hemos estudiado, en menos elementos de los que se esperaba al inicio del estudio, pero ha quedado demostrado que hay elementos de la L2 que obstaculizan el aprendizaje del español:

Imagen 3 - Interferencia lingüística del inglés en la enseñanza de ELE a chinos

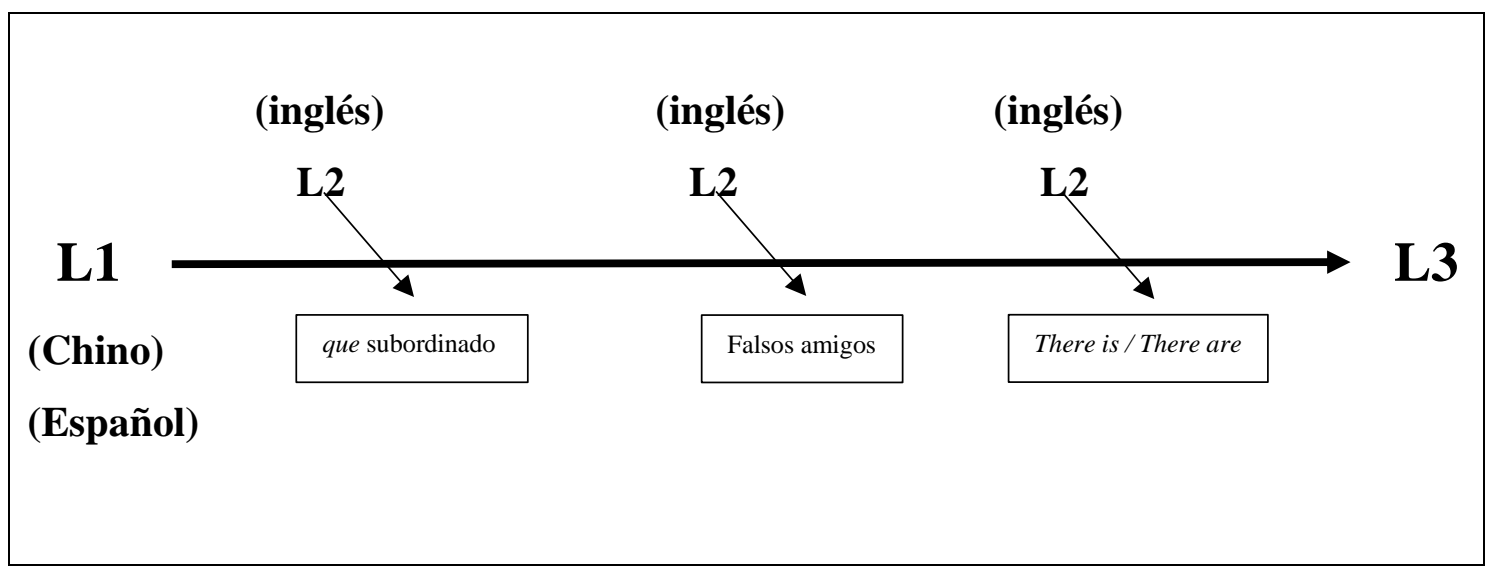

Además de estas influencias negativas del inglés, se han encontrado algunos casos que parecen responder a la interferencia con la lengua materna (el chino mandarín). Estas interferencias se pueden ver en ambas lenguas (inglés y español) o solo en la menos controlado (en este caso, será el español).

También se han encontrado errores que son interferencia del español en las producciones en inglés. En estos casos, la L3 actúa como interferencia en la L2. En la encuesta estos fallos parecen ser mayoría. Esto puede deberse a que estos estudiantes están en un contexto de inmersión lingüística en beneficio de la L3, en el que apenas usan la L2, por lo que puede parecer lógico que esta se vea alterada por el español, pues es la LE que más presente estaba en ese momento en su rutina diaria. No obstante, se 
pueden apreciar las interferencias del inglés en el español, como ya hemos visto, que son mucho más evidentes en las redacciones y en la mayoría de producciones orales de los estudiantes sinohablantes, corpus (el oral) que queda por estudiar en futuras investigaciones.

\section{REFERENCIAS}

ALONSO GARCÍA, Nuria. Descripción de los errores detectados en la interpretación de los equivalentes españoles de la forma inglesa -ING. RESLA, v. 16, p. 7-21, 2003.

CASAS, Helena; ROVIRA, Sara; SUÁREZ, Anne-Hélène. Lengua china para traductores. v. 1 y 2. Bellaterra: Servei de Publicacions de la Universitat Autònoma de Barcelona. 2015.

CORTÉS MORENO, Maximiano. Dificultades lingüísticas de los estudiantes chinos en el aprendizaje del ELE. Carabela, 52. p. 77-98, 2002. Madrid: SGEL. Disponível em: http://203.68.184.6:8080/dspace/handle/987654321/760.

CORTÉS MORENO, Maximiano. Chino y español: un análisis contrastivo. Em: SÁNCHEZ GRUÑÁN, Alberto; MELO, Mónica (Coord.). Qué saber para enseñar a estudiantes chinos. Buenos Aires (Argentina): Voces del Sur, p. 173-200, 2009.

CORTÉS MORENO, Maximiano. Dificultades lingüísticas del español para los estudiantes sinohablantes y búsqueda de soluciones motivadoras. La enseñanza del español para sinohablantes en contextos, n. 10, p. 173-208, 2014.

PO-CHING, Yip; RIMMINGTON. Gramática de la lengua china. Madrid: Cátedra, 2015.

ROVIRA ESTEVA, Sara. Lengua y escrituras chinas. Barcelona: Bellaterra, 2017.

SANTOS GARGALLO, Isabel. Lingüística aplicada a la enseñanza-aprendizaje del español como lengua extranjera. Madrid: Arco/Libros, 1999.

SELINKER, Larry. Interlanguage. IRAL, 10, p. 209-231, 1972.

XUN, Liu. El nuevo libro del chino práctico. Beijing: Beijing Language and Culture University Press, 2008. 


\section{Como citar este artículo}

NAVARRO CARRASCOSA, Carles. Interferencia lingüística del inglés en estudiantes chinos de español. El caso de estudiantes universitarios en España. Rev. EntreLínguas, Araraquara, v. 5, n. esp. 1, p. 328-343, jul., 2019. E-ISSN: 2447-3529. DOI: 10.29051/el.v5iesp.1.12988

Submetido em: 01/06/2019

Aprovado em: 30/06/2019

Publicado em: 01/10/2019 